\title{
Improved Parameter Estimation for First-Order Markov Process
}

\author{
Deepak Batra, ${ }^{1}$ Sanjay Sharma, ${ }^{2}$ and Amit Kumar Kohli ${ }^{2}$ \\ ${ }^{1}$ Department of Electronics and Communication Engineering, CITM, Faridabad 121002, India \\ ${ }^{2}$ Department of Electronics and Communication Engineering, Thapar University, Patiala 147004, Punjab, India
}

Correspondence should be addressed to Amit Kumar Kohli, akkohli@thapar.edu

Received 18 June 2009; Accepted 20 July 2009

Recommended by A. G. Constantinides

This correspondence presents a linear transformation, which is used to estimate correlation coefficient of first-order Markov process. It outperforms zero-forcing (ZF), minimum mean-squared error (MMSE), and whitened least-squares (WTLSs) estimators by controlling output noise variance at the cost of increased computational complexity.

Copyright ( 2009 Deepak Batra et al. This is an open access article distributed under the Creative Commons Attribution License, which permits unrestricted use, distribution, and reproduction in any medium, provided the original work is properly cited.

\section{Introduction}

Let us consider a linear multi-input multioutput model with an unknown $N \times 1$ parameter vector $\vec{x}(n)=$ $\left[a_{1} a_{2} \cdots a_{N}\right]^{T}$, such that

$$
\vec{y}=H \vec{x}+\vec{v}
$$

where, $\vec{y}(n)=[h(n) h(n-1) \cdots h(n-M+1)]^{T}$ is $M \times 1$ output vector,

$H(n)=\left[\begin{array}{cccc}h(n-1) & h(n-2) & \cdots & h(n-N) \\ h(n-2) & h(n-3) & \cdots & h(n-N-1) \\ h(n-M) & h(n-M-1) & \cdots & h(n-N-M+1)\end{array}\right]$

is $M \times N$ complex matrix, and $\vec{v}(n)=[v(n) v(n-1) \cdots$ $v(n-M+1)]^{T}$ is white Gaussian process noise $M \times 1$ vector with zero-mean and covariance matrix $\sigma_{v}^{2} I_{M}$, where $I_{M}$ is $M \times M$ identity matrix. The estimated unknown parameter vector may be defined as $\hat{x}=T \vec{y}$, where " $T$ " is linear transformation involving pseudoinverse. The application of ZF linear transformation $T_{\mathrm{ZF}}=\left(H^{H} H\right)^{-1} H^{H}$ to $\vec{y}$ results in nonwhite noise with covariance matrix $C_{\mathrm{ZF}}=\sigma_{v}^{2}\left(H^{H} H\right)^{-1}$. On the other hand, MMSE linear transformation $T_{\mathrm{MMSE}}=$ $\left(H^{H} H+\sigma_{v}^{2} I_{N}\right)^{-1} H^{H}$ alleviates output noise variance by finding the optimum balance between data detection and noise reduction [1]. However, the modification of least squares estimation is based on the concept of MMSE whitening; that is, WTLS performs well at low to moderate signalto-noise ratios by using linear transformation $T_{\text {WTLS }}=$ $B\left(H^{H} H\right)^{-1 / 2} H^{H}[2]$, where $B=\operatorname{diag}\left[\beta_{1}, \beta_{2}, \ldots, \beta_{N}\right]$ with $\beta=\beta_{1}=\beta_{2}=\cdots=\beta_{N}=\operatorname{Tr}\left\{\left(H^{H} H\right)^{1 / 2}\right\} / \operatorname{Tr}\left\{H^{H} H\right\}$. It follows that

$$
\hat{x}_{\mathrm{WTLS}}=B\left(H^{H} H\right)^{1 / 2} \vec{x}+B\left(H^{H} H\right)^{-1 / 2} H^{H} \vec{v} .
$$

Substitution of the unique QR-decomposition $H=Q D \bar{M}$ in (3) leads to

$$
\hat{x}_{\mathrm{WTLS}}=B D \bar{M} \vec{x}+B Q^{H} \vec{v}
$$

where, $Q=\left[q_{1}, q_{2}, \ldots, q_{N}\right]$ is an $M \times N$ matrix with orthonormal columns, $D$ is an $N \times N$ real diagonal matrix whose diagonal elements are positive, and $\bar{M}$ is an $N \times$ $N$ upper triangular matrix with ones on the diagonal (on contrary to [3]). Incorporation of $B D=I_{N}$ in (4) yields $\hat{x}_{\mathrm{WTLS}}=\bar{M} \vec{x}+D^{-1} Q^{H} \vec{v}$, where $D^{-1} Q^{H}$ is referred to as noise whitening-matched filter [4].

\section{AR(1) Parameter Estimation}

In the presented exposition, the posited linear transformation is $T_{\mathrm{MZF}}=\bar{M}\left(H^{H} H\right)^{-1} H^{H}$. Consequently,

$$
\hat{x}_{\mathrm{MZF}}=T_{\mathrm{MZF}} \vec{y}=\bar{M} \vec{x}+\bar{M}\left(H^{H} H\right)^{-1} H^{H} \vec{v}
$$

with noise covariance matrix $C_{\mathrm{MZF}}=\sigma_{v}^{2} \bar{M}\left(H^{H} H\right)^{-1} \bar{M}^{H}$. This transformation also performs noise whitening. It is 


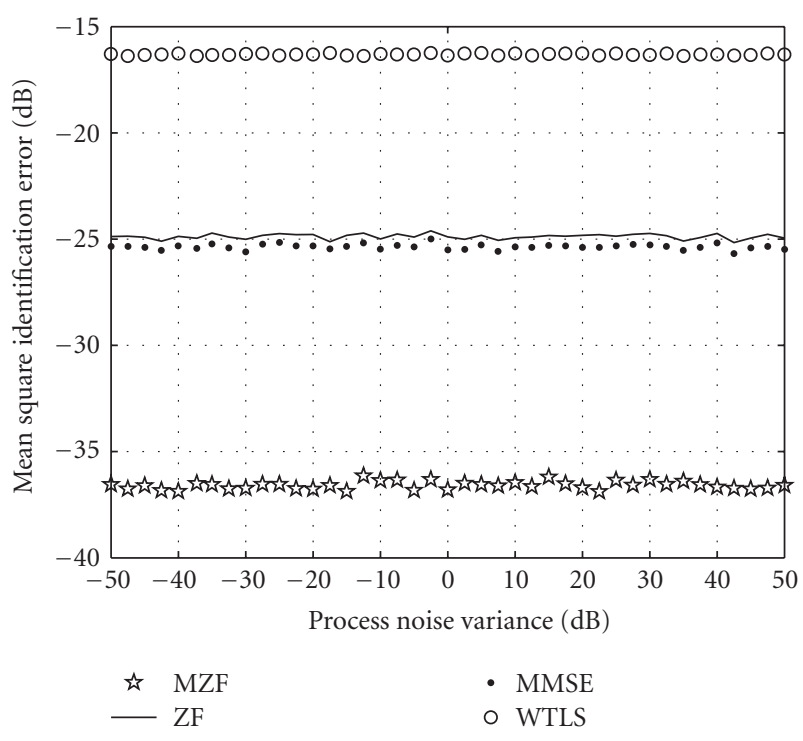

FIGURE 1: MSIE versus process noise variance $\left(\sigma_{v}^{2} \mathrm{~dB}\right)$.

apparent that output noise variance is controlled and reduced, since $0 \leq m_{i, j}<1$ for $i \neq j$ (i.e., $i$ th row and $j$ th column element of matrix $\bar{M})$. For $\operatorname{AR}(1)$ correlation coefficient $\left(a_{1}\right)$ estimation, the unknown parameter vector $\vec{x}$ in (1) and (5) is replaced by $\bar{x}=\left[a_{1} \Delta a_{2} \Delta a_{3} \cdots \Delta a_{N}\right]^{T}$ with leakage coefficients $\Delta a_{j} \rightarrow 0$. Thus, the estimated parametric value is

$$
\hat{x}_{\mathrm{MZF}, 1}=\hat{a}_{1}=a_{1}+\lim _{\Delta a_{j} \rightarrow 0} \sum_{j=2}^{N} m_{1, j} \Delta a_{j}+v_{\mathrm{MZF}, 1}
$$

with $0 \leq m_{1, j}<1$ for $j \neq 1$. For parameter values $a_{1}=0.95$ (true correlation coefficients) and $\Delta a_{2}=\Delta a_{3}=\Delta a_{4} \approx$ 0.0001 (assumed), the simulation results depicted in Figure 1 demonstrate that the proposed technique outperforms other aforementioned linear transformations. However under similar conditions, the value of $\beta$ is found to be high in case of WTLS transform, which in turn increases the output noise variance.

\section{Conclusions}

The presented linear transformation based on a typical QRdecomposition (i.e., $Q D \bar{M}$ ) reduces output noise, which is utilized for the efficient estimation of correlation coefficient in first-order Markov process.

\section{References}

[1] S. Verdú, Multiuser Detection, Cambridge University Press, Cambridge, UK, 1998.

[2] Y. C. Eldar and A. V. Oppenheim, "MMSE whitening and subspace whitening," IEEE Transactions on Information Theory, vol. 49, no. 7, pp. 1846-1851, 2003.

[3] A. K. Kohli and D. K. Mehra, "A two-stage MMSE multiuser decision feedback detector using successive/parallel interference cancellation," Digital Signal Processing, vol. 17, no. 6, pp. 10071018, 2007.
[4] D. W. Waters and J. R. Barry, "Noise-predictive decisionfeedback detection for multiple-input multiple-output channels," IEEE Transactions on Signal Processing, vol. 53, no. 5, pp. 1852-1859, 2005. 

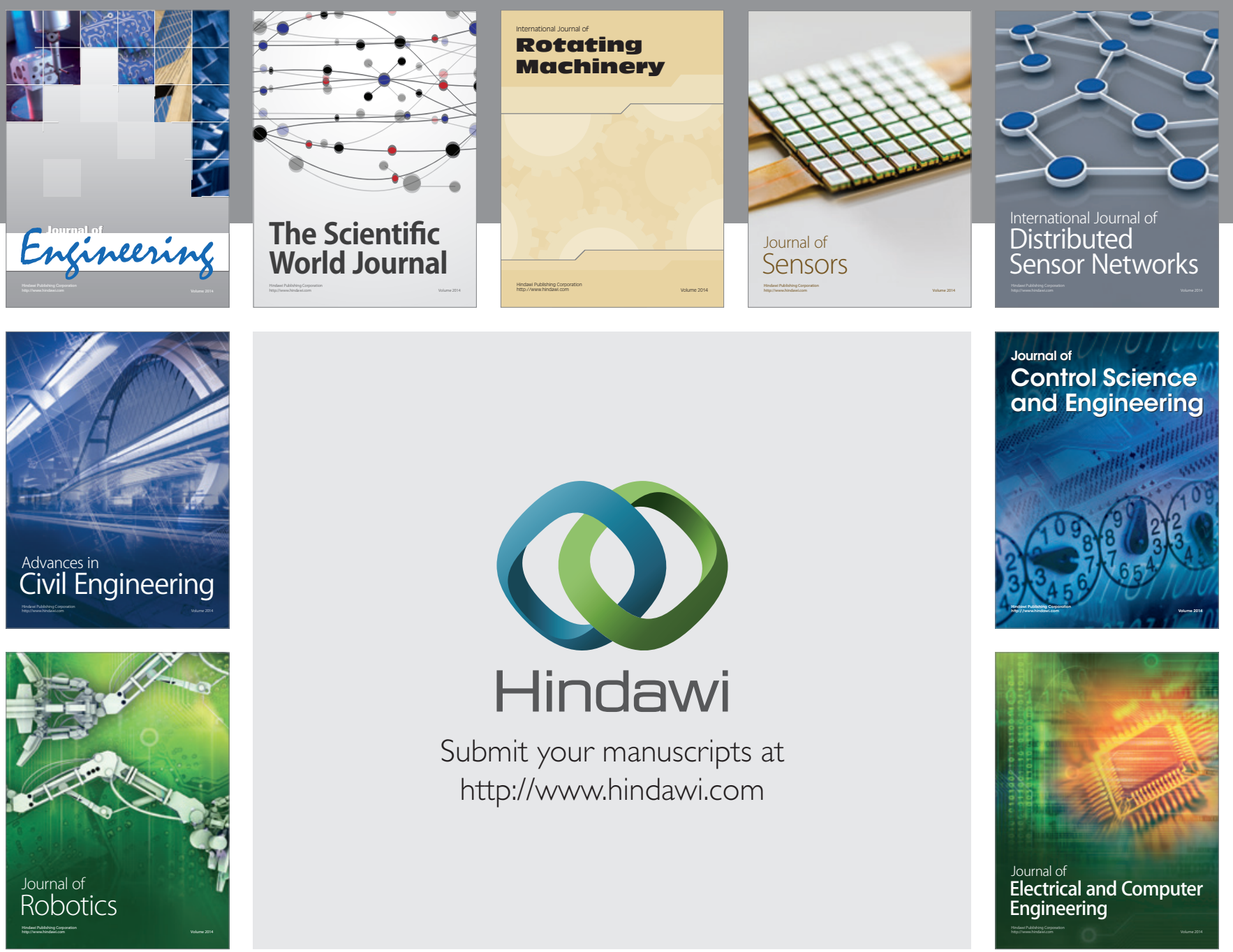

Submit your manuscripts at

http://www.hindawi.com
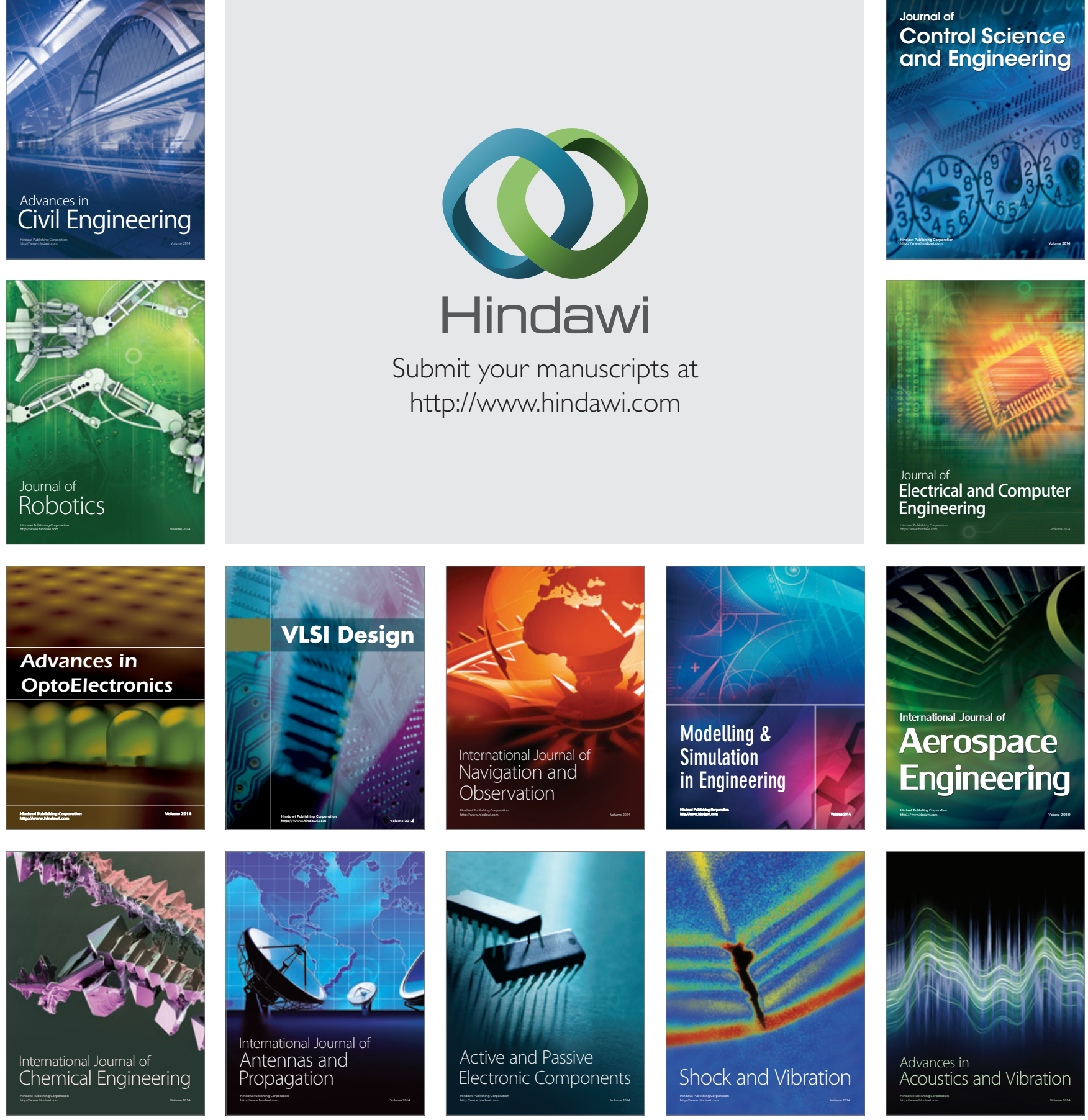